\title{
SPOLM2O19
}

XIX SIMPÓSIO DE PESQUISA OPERACIONAL E LOGISTICA DA MARINHA

RIO DE JANEIRO, RJ, BRASIL - 06 A 08 DE NOVEMBRO DE 2019

\section{Desenvolvimento de Ferramenta Computacional em Python: implementação e estudo de caso dos métodos PROMETHEE I, II e III}

\author{
Miguel Ângelo Lellis Moreira \\ Instituto Militar de Engenharia (IME) \\ Praça Gen. Tibúrcio, 80 - Urca, Rio de Janeiro - RJ, 22290-270 miguellellis@hotmail.- \\ com \\ Marcos dos Santos \\ Instituto Militar de Engenharia (IME) \\ Praça Gen. Tibúrcio, 80 - Urca, Rio de Janeiro - RJ, 22290-270 marcosdossantos_dou- \\ torado_uff@yahoo.com.br

\section{Carlos Francisco Simões Gomes} \\ Universidade Federal Fluminense (UFF) \\ Rua Passos da Pátria, 156, Bloco D, São Domingos, Niterói - RJ, 24210-240 cfsg1@bol.- \\ com.br
}

\section{RESUMO}

O presente artigo objetiva apresentar uma análise explicativa do método de Apoio Multicritério à Decisão PROMETHEE, junto a uma ferramenta computacional para aplicação. Quanto ao método, é exposto as etapas que compõem o processo lógico matemático e os três primeiros métodos de análise da família PROMETHEE. A ferramenta desenvolvida tem o propósito de auxiliar o decisor na aplicação do método, sendo necessário ao usuário apenas inserir os dados requisitados pela aplicação em um dado caso. Um estudo de caso é apresentado para demonstrar o funcionamento da ferramenta e as análises dos resultados obtidos. A ferramenta proposta mostra ser eficaz, podendo ser utilizada em âmbito acadêmico, como forma de auxílio na aprendizagem do método, ou em organizações, como forma de apoio aos gestores em suas tomadas de decisões.

Palavra-chave: Método PROMETHEE; Apoio Multicritério à Decisão; Ferramenta Computacional; Python. 


\begin{abstract}
The present article aims to presente an explonatory analysis of the Multiple Criteria Decision Analysis method PROMETHEE, along with a computational tool for application. With regard to the method, the steps that make up the mathematical logic process and the first three methods of the PROMETHEE Family are exposed. The tool developed has the purpose to assist the decision maker in the method application, being necessary for the user only enter the data requested by the application in a given case. A case study is presented to demonstrate the functioning of the tool and the analysis of the results obtained. The proposed tool proves to be effective and can be applied in academic field, as a form of aid in the learning of the method, or in organizations, as sisting managers in their decision making.
\end{abstract}

Keywords: PROMETHEE Method; Multicriteria Decision Aiding; Computational Tool; Python.

\title{
Como Citar:
}

MOREIRA, Miguel Ângelo Lellis; SANTOS, Marcos dos; GOMES, Carlos Francisco Simões. Desenvolvimento de Ferramenta Computacional em Python: implementação e estudo de caso dos métodos PROMETHEE I, II e III. In: SIMPÓSIO DE PESQUISA OPERACIONAL E LOGÍSTICA DA MARINHA, 19., 2019, Rio de Janeiro, RJ. Anais [...]. Rio de Janeiro: Centro de Análises de Sistemas Navais, 2019.

\section{INTRODUÇÃO}

A tomada de decisão está presente no cotidiano de pessoas e organizações, sempre sendo necessária para o estabelecimento e desenvolvimento de etapas futuras. Tomar uma decisão sempre é necessário quando um sujeito se encontra diante de um problema que possui mais que um meio para o resolver. Mesmo quando há apenas uma escolha para a resolução, o decisor tem a alternativa de tomar ou não a ação como solução (GOMES; GOMES, 2014).

Segundo Costa (2002), uma tomada de decisão pode ser classificada em: Escolha, classificação, ordenação, classificação ordenada e priorização. A mesma também é classificada quanto sua quantidade de critérios utilizados para a análise das alternativas, sendo relacionadas a modelos monocritérios ou multicritérios. Os métodos multicritérios (Multiple-Criteria Decision Method - MCDM, ou Apoio Multicritério à Decisão - AMD) possuem características específicas, trazendo ao decisor uma nova discussão: "Qual alternativa é mais viável para determinadas situações ou requisitos?" (GOMES et al., 2013).

Os métodos multicritério de apoio à decisão, podem ser compreendidos como técnicas que viabilizam a estruturação e análise de problemas complexos de avaliação de forma transparente, com a introdução de critérios quantitativos e qualitativos, em casos específicos, havendo trade-off entre eles. Esses métodos permitem as organizações estruturar um processo decisório, considerando vários aspectos de avaliação, tais como técnicos, socioeconômicos e ambientais por exemplo, em níveis operacionais e estratégicos para tomada de decisão (GRECO; FIGUEIRA; EHRGOTT, 2016).

As aplicações presentes no AMD, possuem técnicas que buscam viabilizar de 
forma mais assertiva, resolver problemas por meio de modelagens matemáticas, auxiliando o decisor na resolução de problemas nos quais podem haver objetivos a serem atendidos de modo simultâneo. Destaca-se que os métodos presentes no AMD não visam apresentar ao decisor uma solução definitiva para a resolução do problema, mas sim apoiar o processo decisório atendendo as restrições requisitadas, dentro de um contexto analisado (MAGALHÃES, 2016)

De acordo com Lehnhart (2016), há um grande número de métodos AMD, portanto nenhum destinado a resolução de todos os tipos de problemas de decisão e sim apropriados em auxiliarem o decisor em um problema específico. Dentre os inúmeros métodos de análise multicritério presentes em âmbito acadêmico, destacamse como os principais, os métodos das escolas americana e francesa. No primeiro caso tem-se o AHP (Analytic Hierarchy Process), trabalhando com análise hierárquicas dos critérios, e o MAUT (Multi-attribute Utility Theory), operando com a teoria de utilidade multiatributo por meio de funções de preferências. Relacionados a escola francesa encontra-se a família de métodos ELECTRE (Elimination and Choice Expressing Reality) e PROMETHEE (Preference Ranking Organization Method for Enrichment Evaluations), operando na ordenação, classificação e seleção de alternativas segundo um dado conjunto de critérios.

Segundo Fontanive (2017), ferramentas computacionais tornam-se facilitadores para análises de decisão multicritério. Por se tratar de modelos matemáticos complexos, o apoio tecnológico viabiliza ao decisor a implementação dos métodos AMD, a partir do momento em que basta ao usuário, compreender seu problema e ter conhecimento dos dados a serem requisitados ao longo do processo decisório.

No estudo apresentado, será dada uma ênfase maior ao método PROMETHEE e suas extensões. 0 método está relacionado aos modelos de relações hierárquicas, em que a ordem preferencial da variável de cada critério analisado é normalizada por uma função de preferência. 0 decisor tem por função indicar informações entre cada critério, no caso de quem deve ser maximizado ou minimizado, e dentro de cada critério, como qual funções de preferência a ser empregada para cada caso (BRANS e SMET, 2016).

0 presente artigo tem por objetivo apresentar uma análise explicativa dos três primeiros métodos pertencentes à família PROMETHEE. Também será apresentado uma ferramenta computacional, desenvolvida em linguagem PYTHON 3.7, destinada a execução do método PROMETHEE em prol da obtenção das ordenações e análises propostas pelas variantes do método I, II e III.

\section{REFERENCIAL TEÓRICO}

Segundo Gonçalo e Alencar (2014), os métodos multicritério têm recebido atenção no campo de ferramentas de apoio à decisão devido à sua robustez e por facilitar a análise de casos mais complexos de forma bastante efetiva. Os métodos AMD surgem como solução viável e objetiva, se apresentando como um conjunto de técnicas matemáticas que auxiliam a análise de tomada de decisão sob influência de um conjunto de critérios (MENDONÇA, 2019)

Gomes e Gomes (2014) dizem que o grande mérito do AMD é conseguir, através de algoritmos e metodologias, possibilitar que a subjetividade seja aceita como parte do processo decisório, auxiliando o tomador de decisão a explicitar confortavelmente suas preferências. 0 desenvolvimento de ferramentas tecnológicas, 
viabiliza a expansão e aplicações dos métodos, a partir do momento que se torna mais simples ao usuário o uso da ferramenta, principalmente quando o decisor não é especialista na área métodos AMD (ISHIZAKA e SIRAJ, 2018).

\subsection{MÉTODOS MULTICRITÉRIO DE APOIO À DECISÃO}

Os principais métodos que compõem o AMD são divididos entre duas grandes escolas, a Escola Americana e a Escola Francesa. Segundo Amorim (2014), a primeira escola, sendo pioneira na linha de pesquisa em tomada de decisão multicritério, baseada na teoria da utilidade, utilizando métodos caracterizados pelo auxílio ao decisor na formulação da função utilidade representativa das suas preferências. A escola Francesa consiste no estudo de métodos de subordinação não compensatórias em um conjunto finito de alternativas, obtendo uma relação de dominância entre elas e resultando em uma ordenação, classificação ou seleção (GOMES e COSTA, 2015).

0 método AHP (Analytic Hierarchy Process), foi apresentado por Saaty (1980) para auxiliar a tomada de decisão em cenários constituídos por vários critérios. 0 método é utilizado em processos de análise decisória estabelecendo prioridades entre alternativas expostas a um conjunto de critérios, considerando aspectos quantitativos e qualitativos da decisão (BOGDANOVIC,2011).

Relacionado ao método ELECTRE, introduzido por Benayoun et al. (1966), o modelo é adequado a situações em que o decisor quer incluir diferentes critérios e pode haver uma coleção robusta associada com a natureza de avaliação envolta em vários padrões (ADEEL et al., 2019). Os métodos que compõem a família ELECTRE baseiam-se em uma lógica não compensatória estabelecendo relações de superação entre pares de alternativas (NEPOMUCENO e COSTA, 2015)

Os métodos multicritério da família PROMETHEE, proposto por Brans et al. (1984), foco deste estudo, têm segundo Vinckle (1992), o objetivo de construir relações de sobreclassificação de valores em problemas de decisão. Carvalho e Curi (2016) afirmam que as principais características do método PROMETHEE são sua simplicidade, clareza e estabilidade, onde a noção de critério generalizado é usada na construção de uma relação valorada de sobreclassificação. O PROMETHEE estabelece uma estrutura de preferência entre as alternativas discretas, tendo uma função de preferência entre as alternativas para cada critério (ARAÚJO \& ALMEIDA, 2009).

Segundo a literatura (BRANS; VINCKE, 1985; BRANS; VINCKE; MARESCHAL, 1986; ALMEIDA; COSTA, 2002), a família PROMETHEE é composto por seis métodos, sendo descritas na sequência: PROMETHEE I: Pré-ordem parcial, resultante da aceitação de uma possível relação de incomparabilidade entre as alternativas; PROMETHEE II: Pré-ordem completa entre as alternativas, designado à problemática de ordenação; PROMETHEE III - Ampliação da noção de indiferença, tratamento probabilístico dos fluxos; PROMETHEE IV - Pré-ordem completa ou parcial, destinada à problemática de escolha e ordenação em situações onde o conjunto de soluções viáveis é contínuo; PROMETHEE V - sendo estabelecida uma ordenação completa entre as alternativas pelo método PROMETHEE II, são introduzidas restrições, requisitadas no problema, para as alternativas selecionadas, elaborando-se uma programação linear inteira para otimização dos resultados; PROMETHEE VI - Pré-ordem completa ou parcial. Utilizada na resolução de problemas de escolha e ordenação. Destinado às situações em que não é possível ao o decisor estabelecer valores fixos quanto aos pesos destinados a cada critério. 


\subsection{PYTHON 3.7}

Python é uma linguagem de programação de alto padrão que combina os paradigmas procedural, funcional e orientado a objetos. A linguagem é muito utilizada por programadores profissionais e é muito utilizada como primeira linguagem em cursos de introdução a programação. A linguagem Python vem se mostrando uma alternativa viável, comparando-a a linguagens mais tradicionais, com $\mathrm{C}, \mathrm{C}++$ e Java por exemplo (JESUS; SANTANA; BITTENCOURT, 2019).

De acordo com Borges (2014), a linguagem inclui diversas estruturas de alto nível (listas, dicionários, complexos etc.), um conjunto de módulos disponíveis para uso e frameworks de terceiros em que a linguagem os permite de serem adicionados. Python é um software de código aberto, com licença compatível com a General Public License.

\section{PROMETHEE}

O PROMETHEE é um método de subordinação que tem por objetivo garantir ao decisor a seleção de um conjunto de alternativas ou ações, sendo avaliadas segundo critérios considerados pelo decisor (BRANS e VINCKE, 1985).

Considerando A um conjunto composto por alternativas, para cada uma, ai $\in$ $A, i=1, \ldots$ n, fj (ai) será uma avaliação dessa alternativa segundo o critério j, j = 1, ... k. Sendo essas avaliações representadas por uma matriz M:

$$
M=\left[\begin{array}{cccc}
\mathrm{f}_{1}\left(\mathrm{a}_{1}\right) & \mathrm{f}_{2}\left(\mathrm{a}_{1}\right) & \ldots & \mathrm{f}_{\mathrm{k}}\left(\mathrm{a}_{1}\right) \\
\mathrm{f}_{1}\left(\mathrm{a}_{2}\right) & \mathrm{f}_{2}\left(\mathrm{a}_{2}\right) & & \mathrm{f}_{\mathrm{k}}\left(\mathrm{a}_{2}\right) \\
\vdots & \vdots & & \\
\mathrm{f}_{1}\left(\mathrm{a}_{\mathrm{n}}\right) & \mathrm{f}_{2}\left(\mathrm{a}_{\mathrm{n}}\right) & & \mathrm{f}_{\mathrm{k}}\left(\mathrm{a}_{\mathrm{n}}\right)
\end{array}\right]
$$

\subsection{FUNCIONALIDADE DO MÉTODO}

O método PROMETHEE pode ser iniciado a partir da matriz $\mathrm{M}$, sendo realizado uma avaliação aos pares, gerando um grau de preferência, por exemplo, da alternativa $\mathrm{a}_{1}$ sobre a alternativa $\mathrm{a}_{2}$ para o conjunto de critério $\mathrm{j}$.

\subsubsection{Função de Preferência Generalizada}

Para cada critério definido é necessário especificar uma função de preferência generalizada $\left(\mathrm{P}_{\mathrm{j}}\right)$ tal que:

$$
\mathrm{Pj}: \mathrm{A} \times \mathrm{A} \rightarrow[0,1]
$$

Comparando as alternativas $a_{1}$ e $a_{2}$, pertencentes ao conjunto $A$, tem-se:

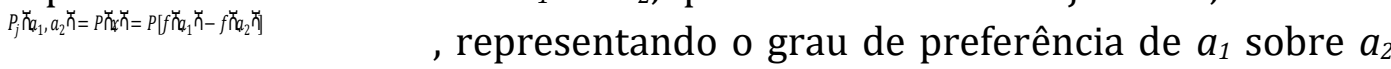
segundo o critério $j$. Sendo assim:

- $\mathrm{P}(\mathrm{x})=0, a_{1}$ não é preferível em relação a $a_{2}$;

- $\mathrm{P}(\mathrm{x}) \cong 0, a_{1}$ é pouco preferível em relação a $a_{2}$;

- $\mathrm{P}(\mathrm{x}) \cong 1, a_{1}$ é fortemente preferível em relação a $a_{2}$;

- $\mathrm{P}(\mathrm{x})=1, a_{1}$ é totalmente preferível em relação a $a_{2}$.

Quando o critério precisa ser maximizado, usa-se $\mathrm{x}=f\left(\mathrm{a}_{1}\right)-f\left(\mathrm{a}_{2}\right)$ para definição da função de preferência. Caso haja a necessidade de minimização do critério, a função de preferência será dada por $\mathrm{x}=f\left(\mathrm{a}_{2}\right)-f\left(\mathrm{a}_{1}\right)$. 
De acordo com Brans, Vincke e Mareschal (1986) há seis tipos de funções de preferência generalizadas, não sendo exaustivo a essas, porém satisfazem a maioria dos casos de aplicações práticas. As funções são definidas na tabela a seguir:

Tabela 1 - Funções de Preferência do método PROMETHEE.

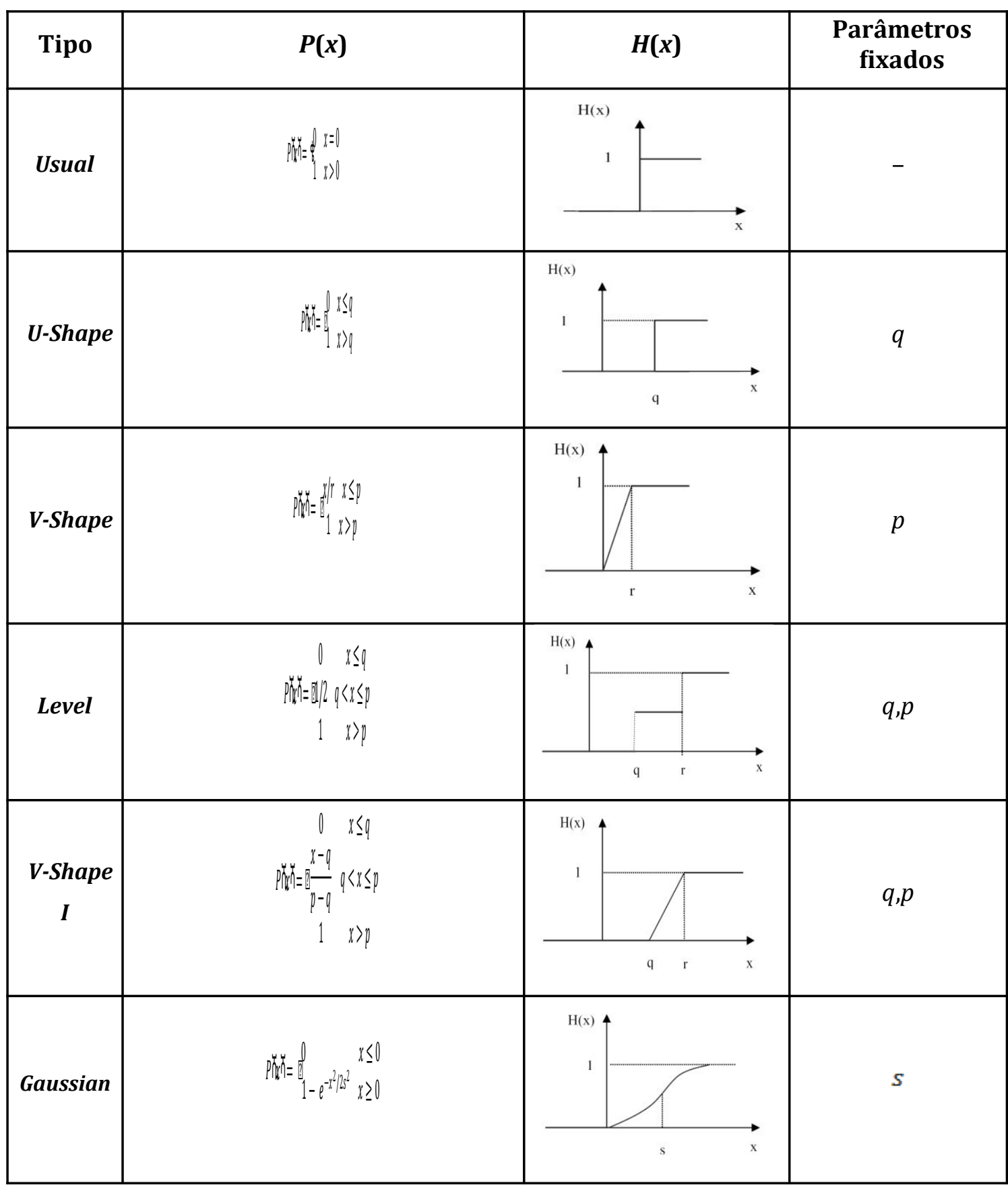

Fonte: (BRANS; VINCKE; MARESCHAL, 1986).

Na função de preferência Usual há indiferença entre as alternativas $a_{1}$ e $a_{2}$ se

Quando as avaliações são diferentes o avaliador tem preferência total pela alternativa de melhor avaliação. 
Na função de preferência U-Shape, as alternativas serão indiferentes, caso não excedam o limite de indiferença $q$. Caso contrário, será dada preferência pela alternativa com melhor avaliação.

A função de preferência $V$-Shape, $p$ é o limite de preferência e, se a diferença entre as alternativas for menor que esse limite, a preferência pela alternativa $a_{1}$ quanto $a_{2}$ aumenta linearmente com a diferença $x$. Quando a diferença é maior que $p$, há total preferência pela alternativa $a_{1}$.

$\mathrm{Na}$ função de preferência Level, o avaliador define o limite de indiferença $q$ e o limite de preferência $p$, tal que: sendo $x$ menor que $q$, haverá indiferença entre as alternativas. Se $x$ está entre $q$ e $p$, há fraca preferência por $\mathrm{a}_{1}$ quanto $a_{2}$, e se $x$ é maior que $p, a_{1}$ é preferível à $a_{2}$.

Na função de preferência $V$-Shape $I$ o avaliador considera que sua preferência aumenta linearmente da indiferença para a preferência entre os limites $q$ e $p$.

A função de preferência Gaussian é necessário ao decisor definir o parâmetro $s$, responsável por indicar o valor a partir do qual há mudança na concavidade na curva de preferência.

\subsubsection{Definição de Pesos}

Definida as funções de preferência, é necessário definir os pesos $\alpha \mathrm{j}(\mathrm{j}=1, \ldots, \mathrm{n})$ referentes aos graus de importância destinado a cada critério j. Esta etapa é determinada pelo decisor. Caso os critérios obtenham os mesmos graus te importância, os valores respectivos aos pesos serão todos iguais.

\subsection{3. Índice de Preferência Ponderada Global}

Nesta etapa é calculado para cada par comparado, o índice de preferência ponderada global $\pi\left(\mathrm{a}_{1}, \mathrm{a}_{2}\right)$, indicando o percentual de preferência da alternativa $a_{1}$ em relação a alternativa $a_{2}$, sendo considerado os pesos atribuídos a cada critério definido.

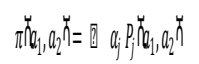

Onde:

$$
0 \leq \pi \mathbb{H}_{1}, Q_{2} \hat{h}_{2} \leq 1 \forall a_{1}, l_{2} \in A
$$

\subsubsection{Fluxo de Importância Positivo}

É caracterizado pela representação da média de preferência de $\mathrm{a}_{1}$ sobre todas as demais alternativas pertencentes ao conjunto A.

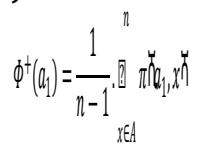

Assim sendo, quanto maior for o fluxo de importância positivo para $\mathrm{a}_{1} \mathrm{C}$ )melhor será a alternativa. 


\subsubsection{Fluxo de Importância Negativo}

Ao contrário do modelo apresentado no item anterior, o fluxo de importância negativo é representado pela média de preferência de todas as alternativas sobre $\mathrm{a}_{1}$.

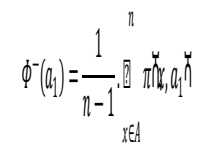

Quanto menor for o fluxo negativo para $\mathrm{a}_{1}(\quad$ ), melhor será a alternativa.

\subsubsection{Fluxo de Importância Líquido}

Definido os fluxos de importância positivo e negativo, será definido o fluxo líquido, com objetivo de gerar os graus de importância para cada alternativa e hierarquiza-las seguindo uma ordem decrescente. Este fluxo é calculado pela diferença encontrada entre os fluxos positivo e negativo, conforme é apresentado a seguir:

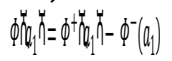

- Onde $\mathrm{a}_{1}$ é preferível à $\mathrm{a}_{2}$

- Onde $\mathrm{a}_{1}$ é indiferente à $\mathrm{a}_{2}$

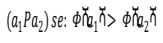

$$
\begin{aligned}
& \left(a_{1} a_{2}\right) s e: \phi \breve{\phi}_{1} \breve{h}_{1}=\phi \breve{h}_{2} \breve{\dagger}
\end{aligned}
$$

\subsection{PROMETHEE I}

O primeiro método de análise do PROMETHEE é classificado como uma préordem parcial das alternativas, sendo obtida por meio dos fluxos de importância positivo e negativo. Normalmente, ambos os fluxos não obtêm os mesmos resultados, logo, o PROMETHEE I é compreendido como o cruzamento entre os fluxos (BRANS e VINCKE, 1985).

Para ser obtido a pré-ordem parcial das alternativas é definia as relações de preferência (P), indiferença (I) e incompatibilidade (R).

- Preferência $\mathrm{a}_{1} P \mathrm{a}_{2}$

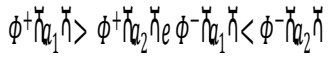

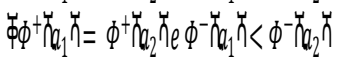

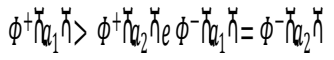

- Indiferença $\mathrm{a}_{1} I \mathrm{a}_{2}$

$$
\phi^{+} \hat{T}_{1} \hat{H}_{1}
$$

- Incompatibilidade $\mathrm{a}_{1} R \mathrm{a}_{2}$

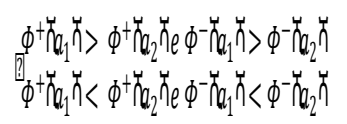

Na relação de preferência, uma força da alternativa $a_{1}$ está associada a uma pequena fraqueza de $a_{1}$ relacionada $a a_{2}$, logo, é considerado que ambos os fluxos, positivo e negativo, são consistentes e podem ser considerados como verdades.

Para a relação de igualdade, ambos os fluxos devem ser iguais para a as alternativas em comparação.

Para que a relação de incompatibilidade seja considerada, é necessário que uma força de $a_{1}$ esteja relacionada a uma fraqueza de $a_{2}$, isso normalmente ocorre quando $\mathrm{a}_{1}$ é bom em um conjunto de critérios que $\mathrm{a}_{2}$ é fraco. Quando isso ocorre, constata que ambos os fluxos obtidos não são consistentes. 


\subsection{PROMETHEE II}

Segundo Brans e Vincke (1985), a extensão PROMETHEE II consiste na utilização das relações de preferência (P) e indiferença (I), utilizando o fluxo líquido de importância obtido pela equação (8), viabilizando gerar uma pré-ordem completa. Neste método, o fluxo líquido pode ser reconhecido como o equilíbrio entre os fluxos positivos e negativos, logo, quanto maior o valor obtido, melhor a alternativa de escolha.

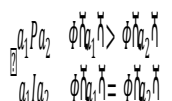

Com a obtenção das relações de preferência, a ordenação será construída de forma decrescente quanto aos valores obtidos no fluxo de importância líquido respectivo a cada alternativa.

\subsection{PROMETHEE III}

Conforme é apresentado por Tzeng e Huang (2011), baseando-se nas razões das extensões anteriores, o PROMETHEE III associa, para cada alternativa a1, um intervalo $\left[x\left(\mathrm{a}_{1}\right), y\left(\mathrm{a}_{1}\right)\right]$, definindo uma pré-ordem completa dos intervalos $(\mathrm{P}, \mathrm{I})$, apresentado na sequência:

$$
\begin{gathered}
a_{1} P a_{2} \text { se } x_{a_{1}}>y_{a_{2}} \\
a_{1} I a_{2} \text { se } x_{a_{1}} \leq y_{a_{2}} \text { e } x_{a_{2}} \leq y_{a_{1}}
\end{gathered}
$$

O intervalo $\left[x\left(\mathrm{a}_{1}\right), y\left(\mathrm{a}_{1}\right)\right]$, é dado por:

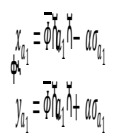

Onde n é reconhecido como o número de critérios:

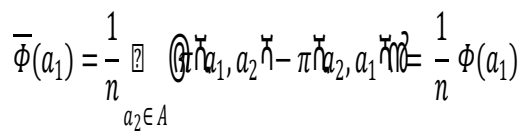

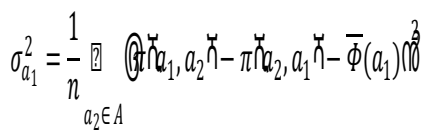

Simplificando $\left[x\left(\mathrm{a}_{1}\right), y\left(\mathrm{a}_{1}\right)\right]$, é um intervalo onde o centro é o fluxo médio líquido de a1 e o comprimento do qual é proporcional ao erro padrão da distribuição dos números $\pi\left(\mathrm{a}_{1}, \mathrm{a}_{2}\right)-\pi\left(\mathrm{a}_{2}, \mathrm{a}_{1}\right)$. Ainda, quanto menor o valor de $\mathrm{a}_{1}$, maior será o valor de superação estrita. Nota-se que o grau de indiferença (I) não seja necessariamente transitivo, enquanto o de preferência (P) ainda é transitivo. Suponha-se três alternativas $a_{1}, a_{2}$ e $a_{3}$, temos $a_{1} I a_{2}$ e $a_{2} I a_{3}$, porém $a_{1} P a_{3}$, conforme é apresentado na figura abaixo:

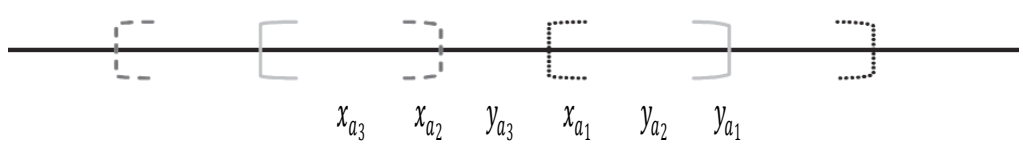

Figura 1 - Relações de a1, a2 e a3. 


\section{EXEMPLO DE APLICAÇÃO}

A ferramenta proposta foi desenvolvida em linguagem Python 3.7, devido a flexibilidade oferecida pela linguagem, viabilizando um algoritmo claro e organizado. 0 código criado possui uma estrutura amigável quanto ao usuário, guiando-o nas etapas de inserção de dados que estruturam a lógica matemática do método PROMETHEE. Para facilitar a execução do script, foi disponibilizado um arquivo executável, sendo apenas necessário ao usuário, abrir o programa em um sistema operacional Windows.

Utilizando como exemplo de aplicação, é apresentado um estudo de caso referente à escolha de um software ERP (Enterprise Resource Planning). No cenário apresentado (Tabela 2), serão analisadas cinco alternativas sendo avaliadas dentro de um conjunto de quatro critérios, são eles: Preço, complexidade, segurança e desempenho. 0 critério preço será representado pelos valores respectivos à aquisição de cada software, os valores estarão em milhares de dólares e este critério deve ser minimizado. Quanto a complexidade, serão registrados em uma escala de 1 a 10 , quanto menor o valor atribuído, menos complexo é o software e este critério deve ser minimizado. A segurança é avaliada em uma escala de 1 a 4, quanto maior o valor registrado, melhor será a alternativa dentro do conjunto e este critério será maximizado. 0 último critério é o de desempenho, os valores são respectivos ao número de transações por hora permitidos em cada software e devem ser maximizados.

Tabela 2 - Matriz de Avaliação para escolha do software.

\begin{tabular}{|c|c|c|c|c|c|c|}
\hline \multirow{2}{*}{$\begin{array}{c}\text { Alternativas } \\
\text { Critérios }\end{array}$} & SARP & ORAC & TOTS & MICRO & IBRP & $\begin{array}{c}\text { Funções } \\
\text { max / min }\end{array}$ \\
\cline { 2 - 7 } Preço & 15 & 29 & 38 & 24 & 25.5 & Min \\
\hline Complexidade & 7.5 & 9 & 8.5 & 8 & 7 & Min \\
\hline Segurança & 1 & 2 & 4 & 3 & 3 & Max \\
\hline Desempenho & 50 & 110 & 90 & 75 & 85 & Max \\
\hline
\end{tabular}

\subsection{EXECUÇÃO DO PROGRAMA PROMETHEE_I_II_III}

O programa é iniciado e em sua tela inicial é apresentado as etapas que estruturam a execução do método PROMETHEE, logo em seguida é solicitado ao usuário a inserção do número de alternativas e critérios que serão avaliados no dado caso.

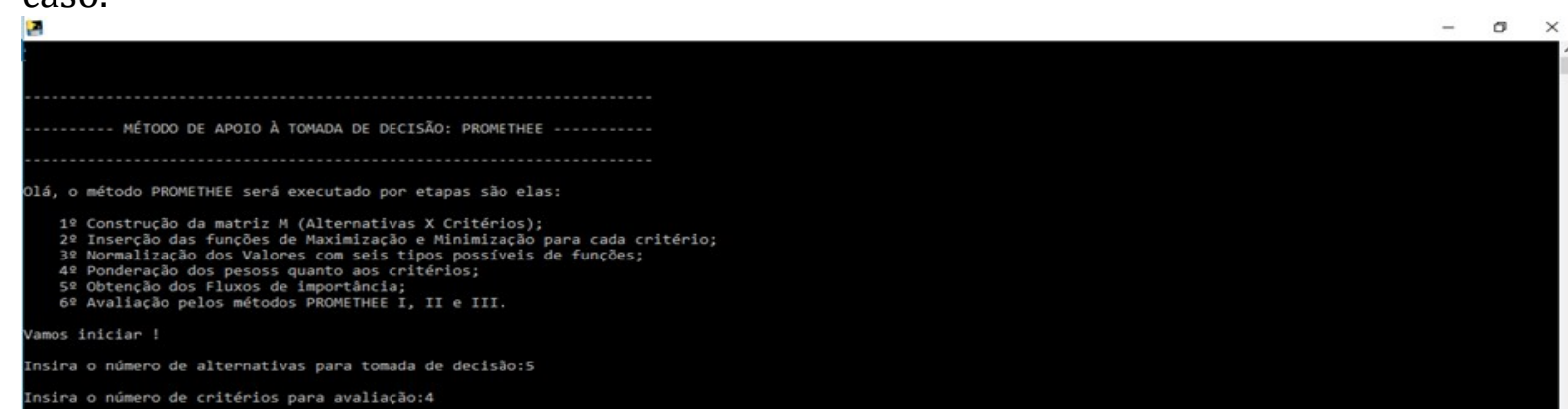

Figura 2 - Tela inicial do Programa PROMETHEE_I_II_III

Conforme apresentado na figura 3, na sequência da execução, são inseridos os nomes das alternativas e dos critérios. Também é apresentado ao usuário a estrutura de matriz de avaliação que será construída na etapa posterior. 


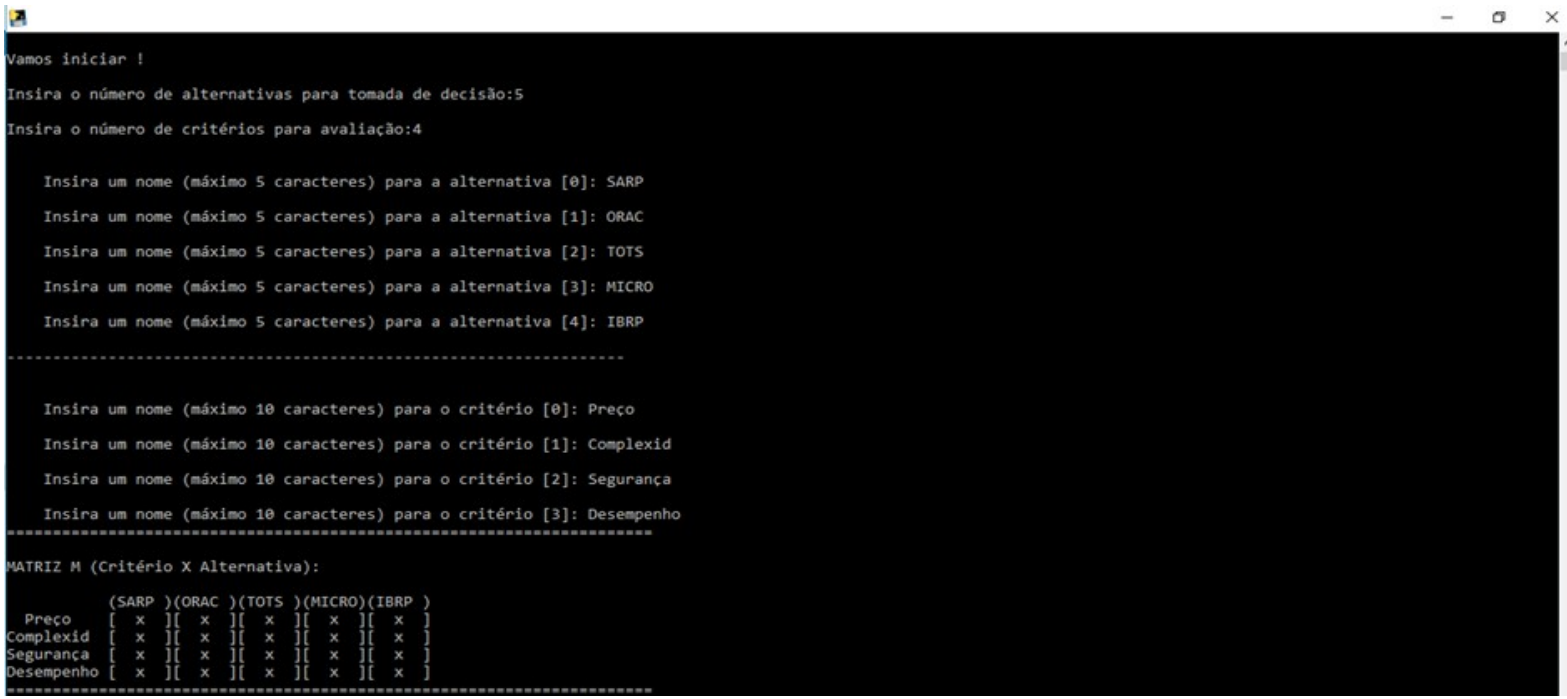

Figura 3 - Tela de nomeação dos dados.

A próxima etapa é destinada a inserção dos dados numéricos respectivos ao conjunto de alternativas em cada critério. Após registrados, esses valores são inseridos e é apresentado a matriz de avaliação completa ao seu respectivo caso.

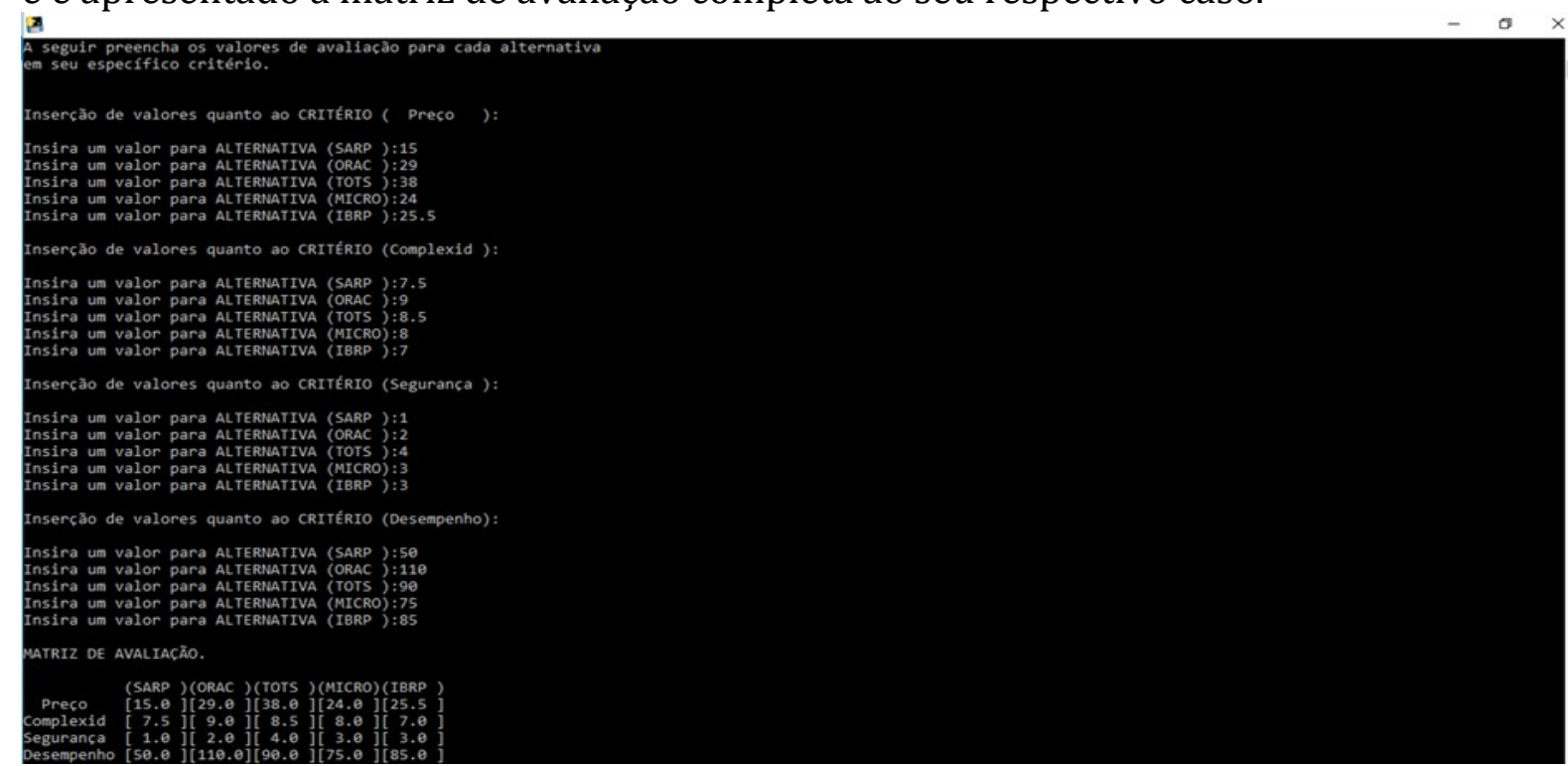

Figura 4 - Tela de inserção de dados quantitativos.

Dentro do método PROMETHEE é necessário ao usuário, expor quais critérios devem ser maximizados ou minimizados. Seguindo esta lógica, na figura 5, para cada critério de avaliação, o decisor deve especificar a função a ser trabalhada digitando "max" para a função de maximizar ou "min" para a função de minimizar. Caso o usuário insira qualquer dado diferente do requisitado, é apresentado uma mensagem de erro, sendo solicitado especificar novamente. 


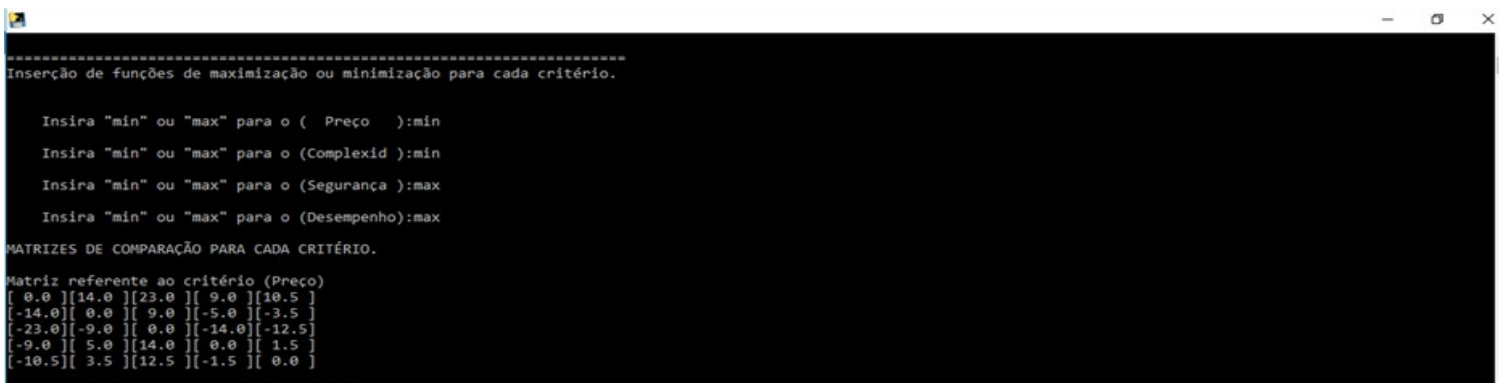

Figura 5 - Tela de especificação para funções de minimizar e maximizar.

Após especificar as funções de comparação, é apresentado uma matriz (Alternativa x Alternativa) para cada critério. Nas matrizes são impressos os resultados das comparações realizadas entre cada par de alternativas, segundo a lógica de maximização e minimização do método trabalhado.

A etapa seguinte é destinada a obtenção das normalizações dos valores. Como apresentado no capítulo anterior, a normalização é viabilizada por meio da utilização de uma das seis funções de preferência para cada critério, cada função com suas respectivas características e parâmetros de avaliação. No estudo de caso, para os quatro critérios foi utilizada a função linear com os parâmetros de indiferença (q) e preferência (p). Os dados são apresentados na tabela a seguir.

Tabela 3 - Funções de Preferência para normalização.

\begin{tabular}{|c|c|c|c|}
\hline \multirow{2}{*}{ Critérios } & \multirow{2}{*}{ Função } & \multicolumn{2}{|c|}{ Parâmetros } \\
\cline { 3 - 4 } & & $\boldsymbol{q}$ & $\boldsymbol{P}$ \\
\hline Preço & V-Shape I & 2 & 5 \\
\hline Complexidade & V-Shape I & 0.5 & 1 \\
\hline Segurança & V-Shape I & 1 & 2 \\
\hline Desempenho & V-Shape I & 10 & 20 \\
\hline
\end{tabular}

Quanto a esta etapa, o sistema apresenta os seis tipos de funções presentes ao método PROMETHEE, junto com os parâmetros que serão solicitados. Cada função é relacionada a um valor numérico de 1 a 6, o usuário digita o valor correspondente e logo após, caso necessário, é solicitado a inserção dos parâmetros de indiferença e preferência. Os valores normalizados são apresentados em uma matriz para cada critério.

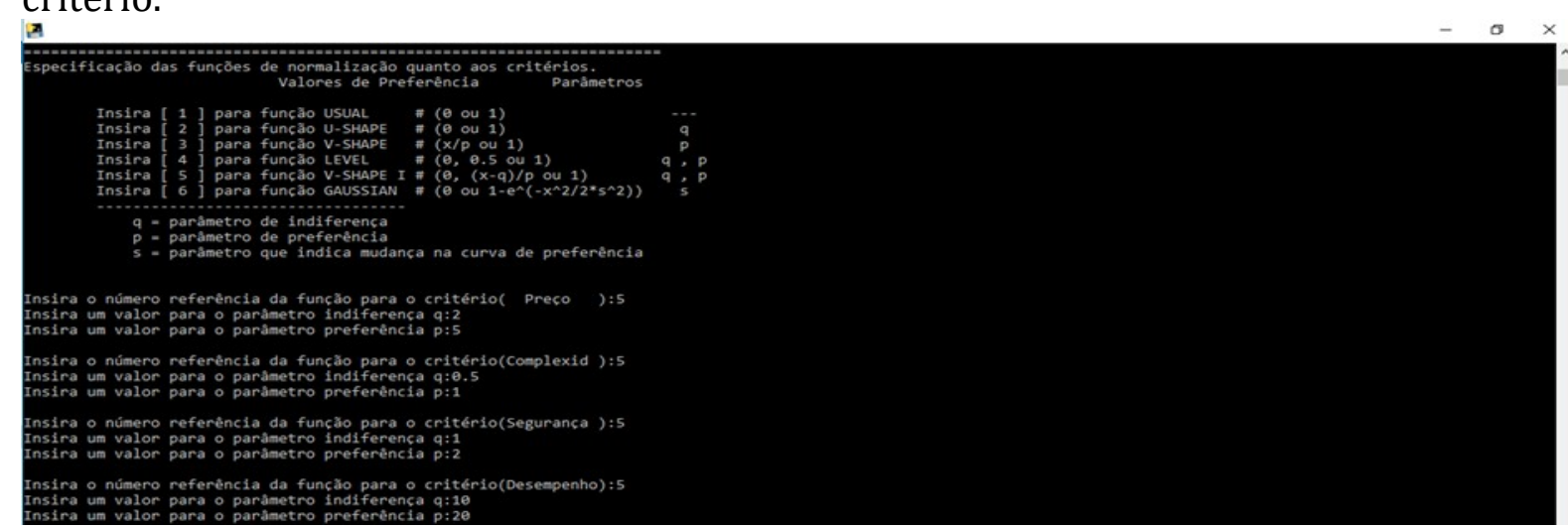

Figura 6 - Tela de especificação de funções para normalização dos valores.

A etapa seguinte é destinada a aplicação dos pesos aos valores normalizados, caso seja o mesmo peso para todos os critérios, basta o usuário digitar a letra "i", caso contrário, digita-se a letra " $\mathrm{P}$ " para o registro dos valores ponderados. Para cada critério é solicitado o valor do peso, que deve estar entre 0,01 e 1 . Ao final, o algoritmo 
faz uma validação da soma dos pesos, caso o resultado seja diferente de 1 , será solicitado uma nova inserção dos pesos.

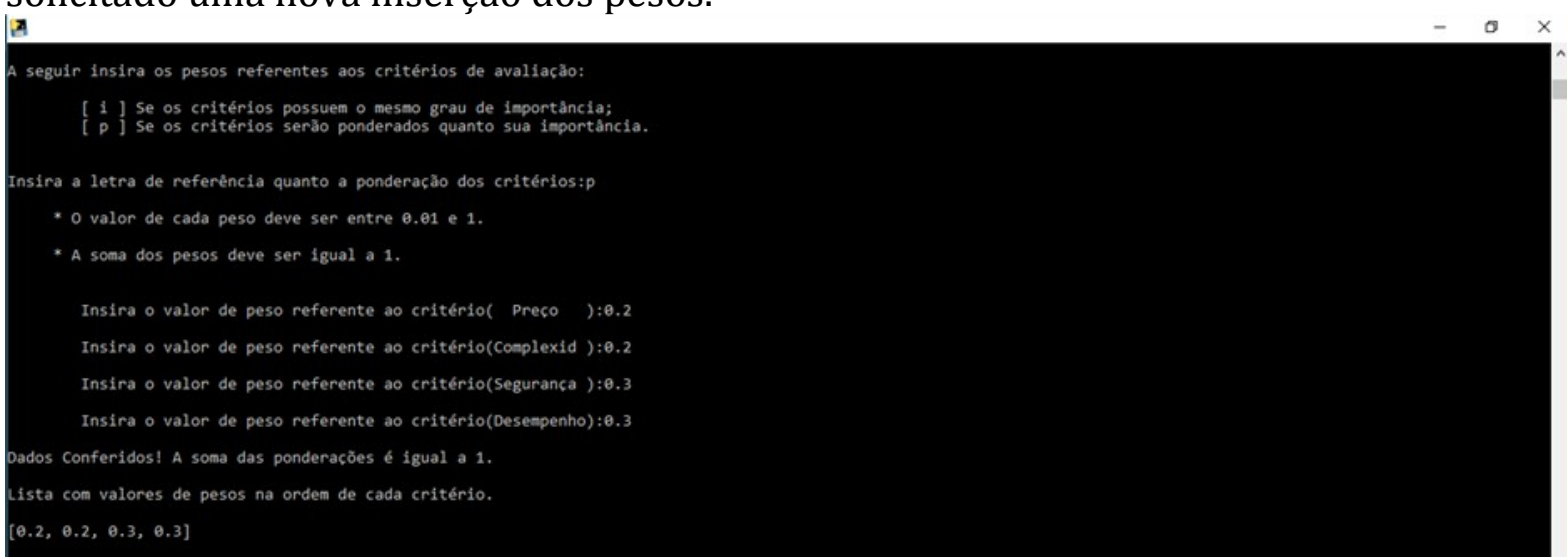

Figura 7 - Tela de inserção de pesos para os critérios.

Finalizando as etapas de inserção de dados, o sistema apresenta inicialmente uma matriz com as médias dos valores obtidos após a aplicação dos pesos. Seguindo a estrutura do método, por meio desses valores, serão obtidos os fluxos positivos, negativos e líquidos para cada alternativa dentro do conjunto de avaliação. Na sequência, o programa apresenta ao decisor três modelos de análise presentes na família de métodos PROMETHEE.

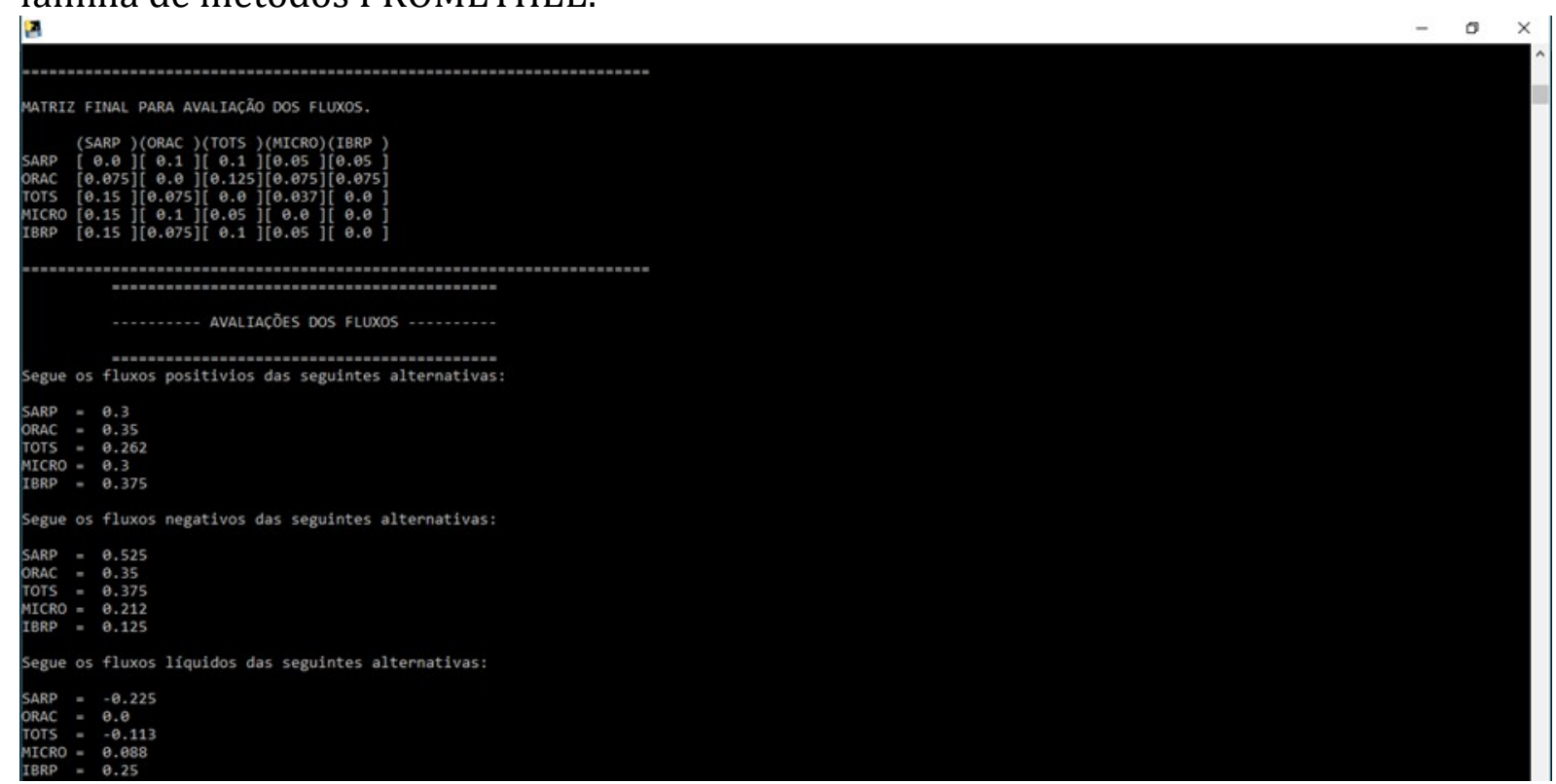

Figura 8 - Tela de resultados com os fluxos de importância.

Na figura 9 é detalhado o método PROMETHEE I, obtido pelos fluxos positivos e negativos de cada alternativa. Para cada alternativa é apresentado a relação de preferência desta quando as demais dentro do conjunto. Relacionado ao dado caso, é possível reconhecer que a alternativa de melhor desempenho é o software IBRP, obtendo uma ordem de preferência total quanto as demais alternativas. 


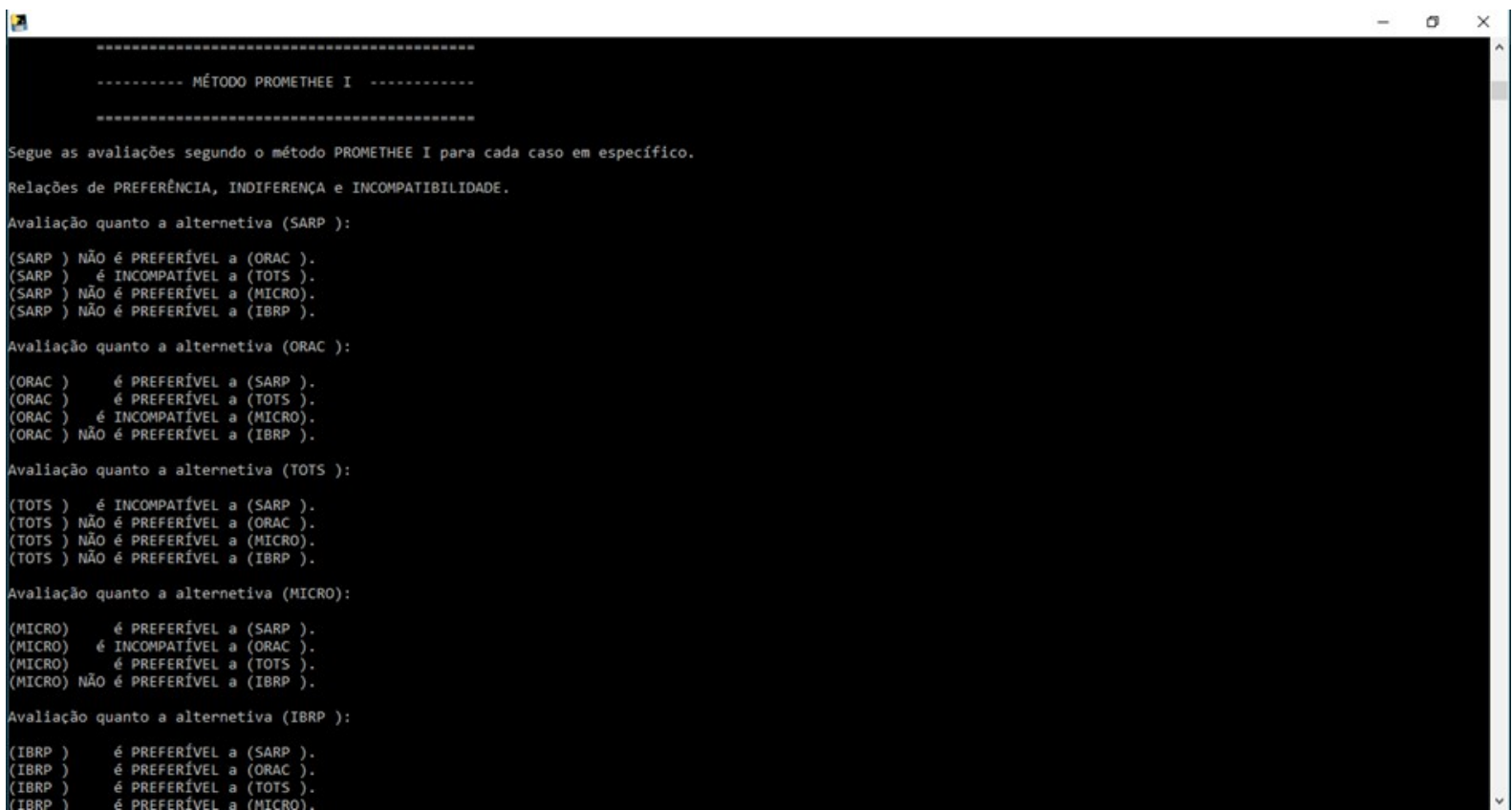

Figura 9 - Tela de análise do método PROMETHEE I.

A análise do método PROMETHEE II, representada pela figura 10, é viabilizada pelos fluxos líquidos de cada alternativa. A impressão dos resultados é apresentada segundo a lógica de ordenação do PROMETHEE II, sendo listado da alternativa mais favorável até a menos favorável quanto a seleção. Nela é possível reconhecer que o software IBRP continua obtendo o melhor desempenho, porém ao contrário do método anterior, é possível analisar de forma mais clara quais são as alternativas com melhores resultados para uma eventual escolha de segunda ou terceira opção.

项

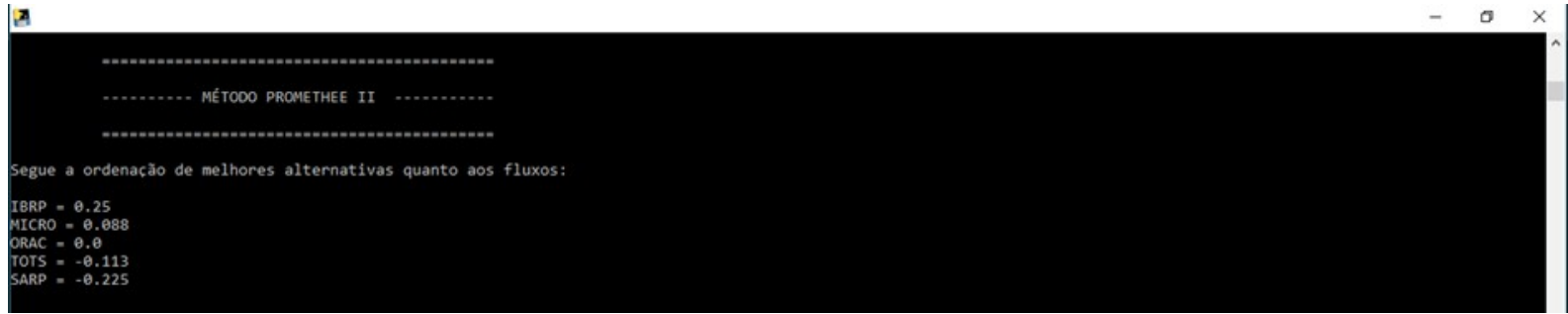

Figura 10 - Tela de análise do método PROMETHEE II.

Na figura 11, referente ao método PROMETHEE III, é apresentado o valor de erro padrão, específico desta variante do método, sendo obtido e listados os valores de limites inferiores (x) e superiores (y) para cada alternativa. Também é exposto as relações de preferências entre todas as alternativas do conjunto.

Quanto ao estudo de caso, o limite inferior do software IBRP foi menor que o limite superior do software MICRO, assim sendo, as duas alternativas passam a ser equivalentes dentro do conjunto de avaliação. 


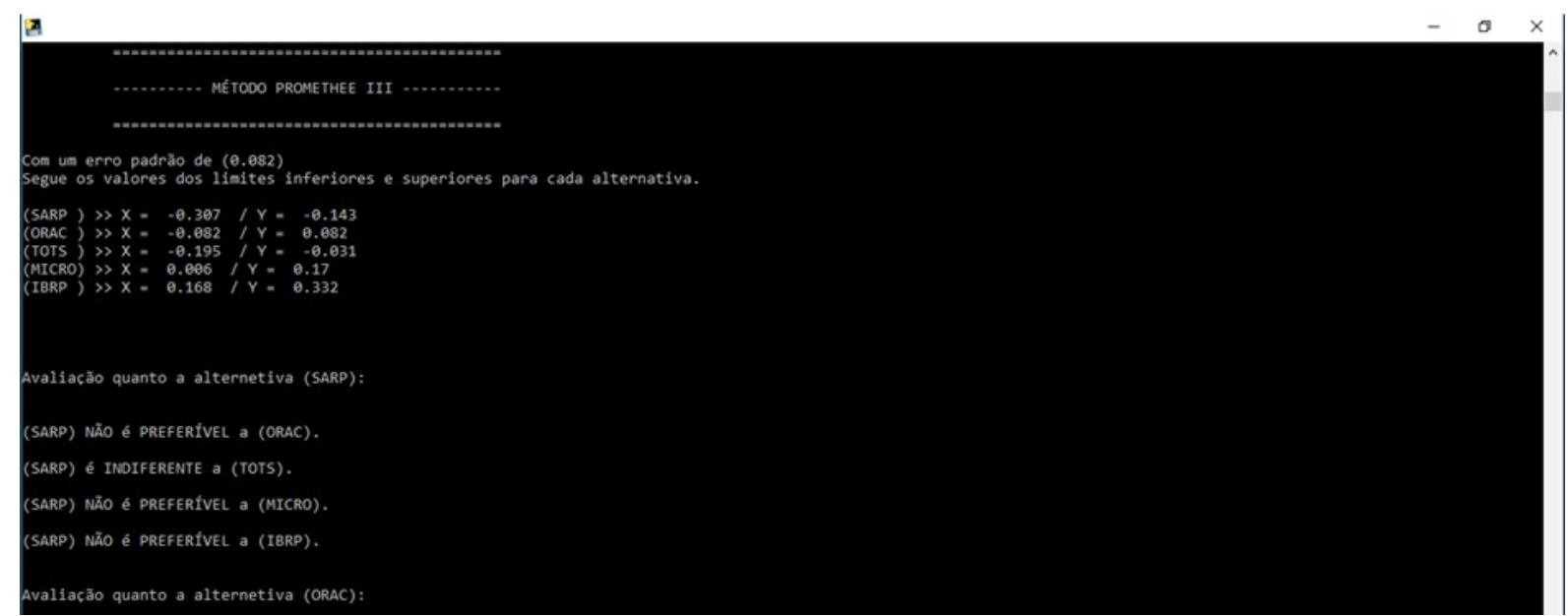

Figura 11 - Tela de análise do método PROMETHEE III.

Dentro do algoritmo proposto, foi viabilizado uma análise gráfica dos resultados. Os gráficos expostos foram adaptados pela utilização da biblioteca Matplotlib, exclusiva do Python. Por meio desta biblioteca foi possível realizar uma adaptação de modelos de gráficos tradicionais, como o gráfico de linhas, para uma análise gráfica que represente as ordenações parciais e completas presentes no método PROMETHEE.

Os gráficos apresentados permitem uma melhor análise de decisão, a partir do momento em que é possível visualizar os três gráficos, respectivos a cada método, de forma simultânea. A imagem dos gráficos pode ser armazenada no computador do usuário para uma análise futura.

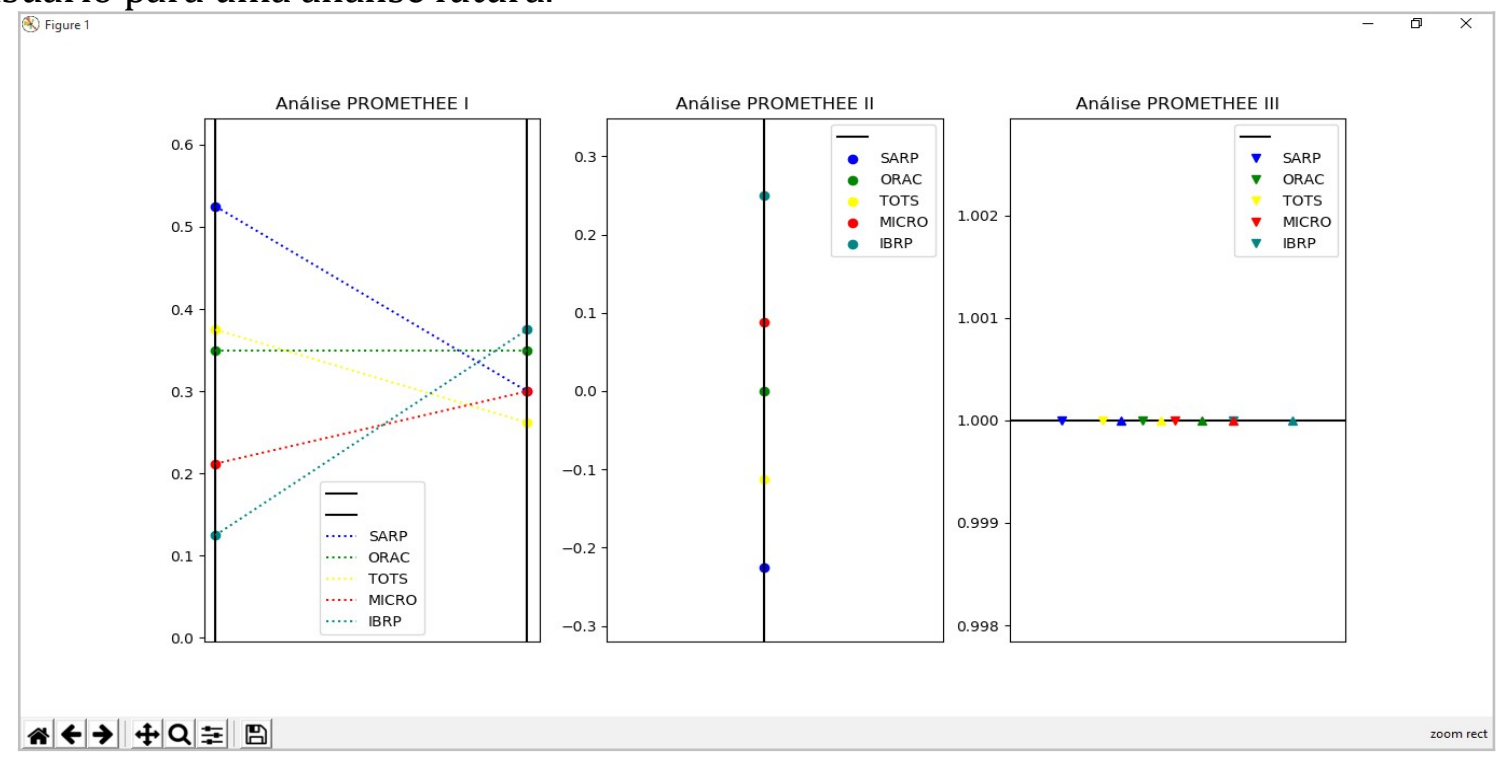

Figura 12 - Tela de análise de análise gráfica dos resultados.

Nesta forma de análise é mais claro a apresentação dos resultados. No primeiro é possível reconhecer que a alternativa IBRP possui o maior fluxo de importância positivo e o menor fluxo negativo, cruzando todas as demais linhas, representando a melhor alternativas dentro do conjunto. 0 segundo gráfico, representação do PROMETHEE II, é mais simples de se analisar, sendo apenas verificado que quanto maior a posição da alternativa, melhor desempenho ela representa. Quanto ao último gráfico, fica claro a relação representada pelos limites inferiores e superiores de cada alternativa, podendo avaliar o quanto determinado software é realmente melhor que o 
outro dentro do conjunto de avaliação.

\section{CONSIDERAÇÕES FINAIS}

0 presente estudo realiza uma análise explicativa do método de apoio à tomada de decisão PROMETHEE. Foi abordado no trabalho as principais particularidades que compõem uma análise decisória multicritério e alguns métodos de apoio, junto a suas características, porém dando ênfase ao método PROMETHEE e suas variantes.

Buscando obter uma melhor compreensão do método, explorou-se todas as etapas do processo que constituem a lógica matemática que estrutura o PROMETHEE, desde a inserção dos dados até a exposição dos resultados. Foi abordado as variantes I, II e III do método original, expondo seus diferentes modelos de análises e manipulações quanto aos fluxos de importância, característicos a cada extensão.

Foi apresentado uma ferramenta computacional, desenvolvida em linguagem Python, para auxiliar o decisor na aplicação do método a um dado caso. 0 algoritmo proposto mostra ser eficiente, guiando o usuário na inserção dos dados, obtenção dos fluxos e ordenação dos resultados, tudo em um formato simples e organizado. 0 conjunto de gráficos apresentado ao final da execução, auxilia o decisor em uma melhor análise visual, sendo possível analisar o quanto determinada alternativa é superior a outra, dentro do conjunto de avaliação.

Como proposta de trabalhos futuros, busca-se estar adaptando o algoritmo desenvolvido para uma aplicação em web. Deste modo, a ferramenta terá uma forma de acesso mais simples, viabilizando auxiliar pessoas e organizações em suas tomadas de decisões.

\section{REFERÊNCIAS BIBLIOGRÁFICAS}

[1] ADEEL, Arooj et al. Novel m-polar fuzzy linguistic ELECTRE-I method for group decisionmaking. Symmetry, v. 11, n. 4, p. 471, 2019.

[2] AMORIM, João Miguel Ramos da Costa. Desenvolvimento de uma ferramenta de apoio à decisão multicritério. Dissertação de Mestrado. Universidade do Minho, Portugal, 2014.

[3] ARAÚJO, Afrânio Galdino de. ALMEIDA, Adiel T. de. Apoio à decisão na seleção de investimentos em petróleo e gás: uma aplicação utilizando o método PROMETHEE. Gest. Prod., São Carlos: 2009.

[4] BENAYOUN, R.; Roy, B.; SUSSMAN, N. Manual de reference du programme electre. Note Synth. Form. 1966.

[5] BOGDANOVIC, Dejan; NIKOLIC, Djordje; ILIC, Ivana. Mining method selection by integrated AHP and PROMETHEE method. Anais da Academia Brasileira de Ciências, v. 84, n. 1, p. 219-233, 2012.

[6] BORGES, Luiz Eduardo. Python para desenvolvedores: aborda Python 3.3. Novatec Editora, 2014.

[7] BRANS, J.; VINCKE, Ph. A preference ranking organisation method: The PROMETHEE method forMCDM. Management Science, v. 31, n. 6, p. 647656, 1985.

[8] BRANS, Jean-Pierre; DE SMET, Yves. PROMETHEE methods. In: Multiple criteria decision analysis. Springer, New York, NY, 2016. p. 187-219.

[9] BRANS, Jean-Pierre; VINCKE, Ph; MARESCHAL, Bertrand. How to select and how to rank projects: The PROMETHEE method. European journal of operational research, v. 24, n. 2, p. 228-238, 1986. 
[10] BRANS, Jean-Pierre; VINCKE, Ph; MARESCHAL, Bertrand. PROMETHEE: A new family of outranking methods in multicriteria analysis. In J.P. Brans, editor, Operational Research '84, pages 477\{490. North-Holland, Amsterdam, 1984.

[11] CARVALHO, J.; CURI, W. F. Sistema de indicadores para a gestão de recursos hídricos em municípios: uma abordagem através dos métodos multicritério e multidecisor. Revista Brasileira de Gestão e Desenvolvimento Regional, v. 12, n. 2, 2016

[12] COSTA, Helder Gomes. Introdução ao Método de Análise de Análise Hierárquica: análise multicritério no auxílio à decisão. Universidade Federal Fluminense, UFF, Niterói, 2002.

[13] FONTANIVE, Fernanda et al. Aplicação do Método de Análise Multicriterial AHP como Ferramenta de Apoio a Tomada de Decisão. Revista Espacios, v. 38, n. 2003, p. 6, 2017.

[14] GOMES, Carlos Francisco Simões; COSTA, Helder Gomes. Aplicação de métodos multicritério ao problema de escolha de modelos de pagamento eletrônico por cartão de crédito. Production, v. 25, n. 1, p. 54-68, 2015.

[15] GOMES, Carlos Francisco Simões; COSTA, Helder Gomes; DE SOUZA, Gabriel Gonzaga. Abordagem estratégica para a seleção de sistemas erp utilizando apoio multicritério à decisão. Revista Produção Online, v. 13, n. 3, p. 1060-1088, 2013.

[16] GOMES, L. F. A. M.; GOMES, C. F. S. Tomada de decisão gerencial: enfoque multicritério. 5 ed. São Paulo: Atlas, 2014.

[17] GONÇALO, Thomas Edson Espíndola; ALENCAR, Luciana Hazin. A supplier selection model based on classifying its strategic impact for a company's business results. Pesquisa Operacional, v. 34, n. 2, p. 347-369, 2014.

[18] GRECO, Salvatore; FIGUEIRA, José; EHRGOTT, Matthias. Multiple criteria decision analysis. New York: Springer, 2016.

[19] ISHIZAKA, Alessio; SIRAJ, Sajid. Are multi-criteria decision-making tools useful? An experimental comparative study of three methods. European Journal of Operational Research, v. 264, n. 2, p. 462-471, 2018.

[20] JESUS, Camille; SANTANA, Bianca; BITTENCOURT, Roberto. Oficinas de Aprendizagem de Programação com Scratch e Python em um Curso de Engenharia de Computação. In: Anais do XXVII Workshop sobre Educação em Computação. SBC, 2019. p. 31-40.

[21] LEHNHART, Eliete dos Reis et al. Tomada de decisão em contextos específicos: uma análise das relações entre os métodos multicritérios de apoio à decisão e as estratégias de decisão humana. 2016. Tese de Doutorado. Universidade Federal de Santa Maria.

[22] MAGALHÃES, Elionai de Souza. Um modelo computacional baseado em análise de decisão multicritério para priorização na alocação de recursos: uma aplicação ao caso das comunidades ribeirinhas da cidade de Coari-AM. 2016.

[23] MENDONÇA, Matheus de Melo et al. Apoio multicritério à decisão para o gerenciamento do sortimento de produtos: um estudo de caso em uma rede de supermercados em Mossoró-RN. 2019.

[24] NEPOMUCENO, Lívia Dias de Oliveira; COSTA, Helder Gomes. Analyzing perceptions about the influence of a master course over the professional skills of its alumni: A multicriteria approach. Pesquisa Operacional, v. 35, n. 1, p. 187-211, 2015.

[25] SAATY, T.L. 1980. The Analytical Hierarchy Process. Mc-Graw-Hill. New York.

[26] VINCKE, Philippe. Multicriteria decision-aid. John Wiley \& Sons, 1992. 